Supporting Information for: Synthetic circuit-driven expression of heterologous enzymes for disease detection

Authors: Jiang $\mathrm{He}^{1,2 \dagger}$, Lior Nissim ${ }^{3,4 \dagger}$, Ava P. Soleimany ${ }^{1,2,5,6 \dagger}$, Adina Binder-Nissim ${ }^{3,7}$, Heather E. Fleming ${ }^{1,2}$, Timothy K. Lu ${ }^{3,8}$, Sangeeta N. Bhatia ${ }^{1,2,8-13 *}$

\title{
Affiliations:
}

${ }^{1}$ Koch Institute for Integrative Cancer Research, Massachusetts Institute of Technology, Cambridge, MA, USA, 02139.

${ }^{2}$ Harvard-MIT Division of Health Sciences and Technology, Institute for Medical Engineering and Science, Massachusetts Institute of Technology, Cambridge, MA, USA, 02139.

${ }^{3}$ Synthetic Biology Group, Research Laboratory of Electronics, Department of Biological Engineering, Massachusetts Institute of Technology, Cambridge, MA, USA, 02139.

${ }^{4}$ Department of Biochemistry and Molecular Biology, The Institute for Medical Research Israel-Canada, Hadassah Medical School, The Hebrew University of Jerusalem, Jerusalem, Israel, 91120.

${ }^{5}$ Harvard Graduate Program in Biophysics, Harvard University, Boston, MA, USA, 02115.

${ }^{6}$ Microsoft Research New England, Cambridge, MA, USA, 02142.

${ }^{7}$ Department of Family Medicine, Meuhedet Health Maintenance Organization, Tel Aviv, Israel, 62038.

${ }^{8}$ Department of Electrical Engineering and Computer Science, Massachusetts Institute of Technology, Cambridge, MA, USA, 02139.

${ }^{9}$ Department of Medicine, Brigham and Women's Hospital, Harvard Medical School, Boston, MA, USA, 02115.

${ }^{10}$ Broad Institute of Massachusetts Institute of Technology and Harvard, Cambridge, MA, USA, 02139.

${ }^{11}$ Wyss Institute at Harvard, Boston, MA, USA, 02115.

${ }^{12}$ Ludwig Center at MIT's Koch Institute for Integrative Cancer Research, Cambridge, MA, USA, 02139.

${ }^{13}$ Howard Hughes Medical Institute, Cambridge, MA, USA, 02139.

${ }^{\dagger}$ These authors contributed equally to this work.

*Corresponding author. Email: sbhatia@mit.edu 

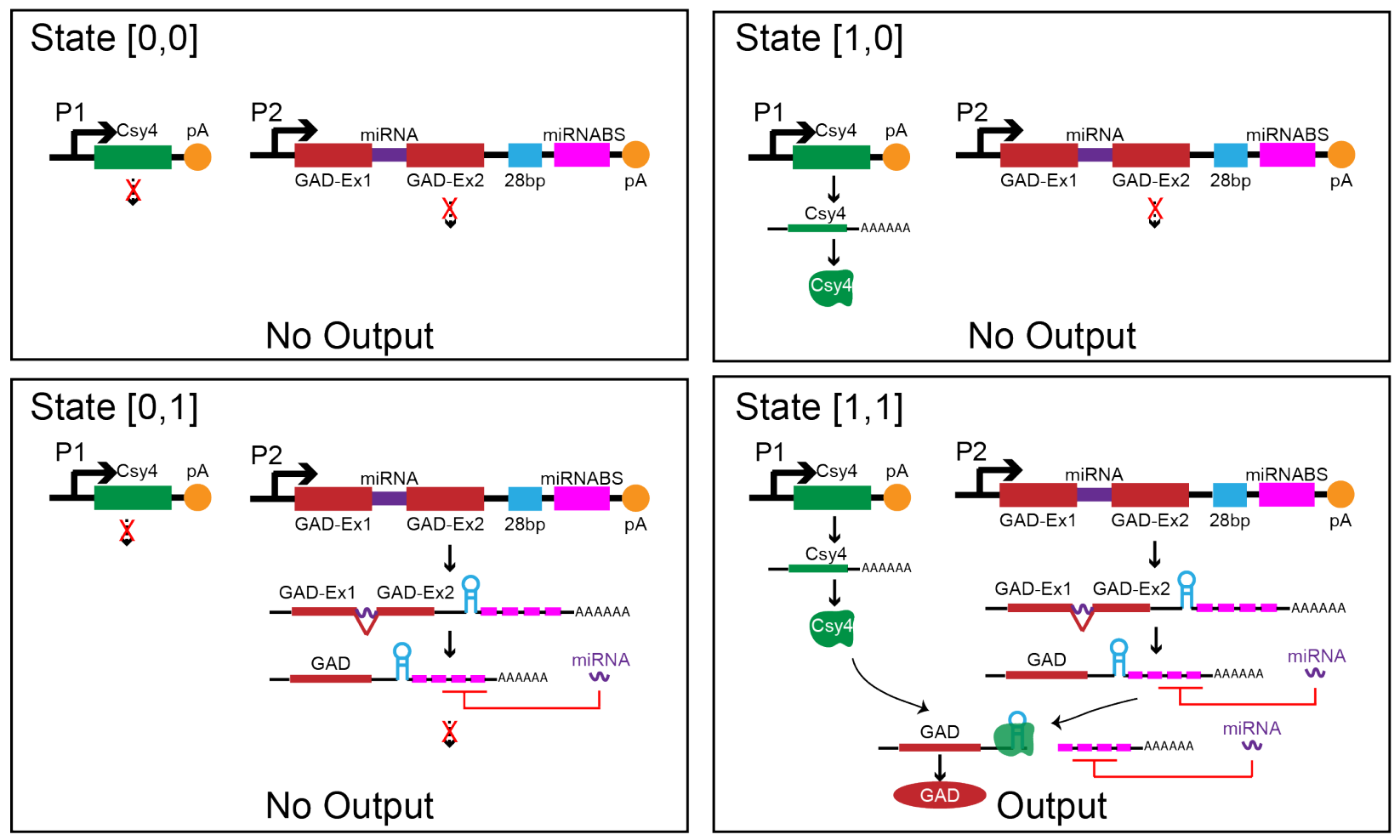

\section{Output Cassette}

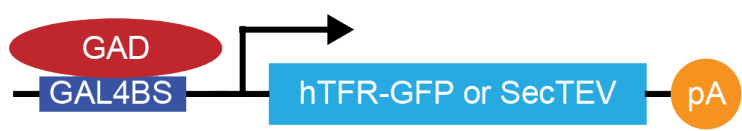

Figure S1. Inputs and states of the RNA/Csy4 based AND gate. In all four states, the output module carrying the output cassette is always present in the circuit. Input states are defined within the square brackets by whether input module 1 and input module 2 are active, where 0 means inactive and 1 means active. In state $[0,0]$, neither $\mathrm{P} 1(\mathrm{~S}(E 2 F 1) \mathrm{P})$ nor $\mathrm{P} 2(\mathrm{~S}(c M y c) \mathrm{P})$ is active, and thus there is no output. In state $[1,0]$, corresponding to when the transcription factor for $\mathrm{S}(E 2 F 1) \mathrm{P}$ but not for $\mathrm{S}(c M y c) \mathrm{P}$ is present, $\mathrm{P} 1$ is active and Csy4 is transcribed. However, as P2 is inactive, no GAD, the fusion protein carrying the GAL4 binding domain and the transcription activator that controls the SPEC on the output expression cassette, will be transcribed; thus, there is no output. In state [0,1], corresponding to when the transcription factor for $\mathrm{S}(c M y c) \mathrm{P}$ but not for $\mathrm{S}(E 2 F 1) \mathrm{P}$ is present, only $\mathrm{P} 2$ is active, and GAD will be transcribed. However, due to the inhibitory effect of the miRNA on the GAD transcript, the transcript cannot be translated; thus there is no output. In state $[1,1]$, both $\mathrm{P} 1$ and $\mathrm{P} 2$ are active. Csy4 will be transcribed to lift the self-inhibitory effect of the miRNA on the GAD transcript, leading to translation of GAD. Only in this state will the output be generated, driven GAD transcriptional activation of the GAL4BS synthetic promoter via active GAL4BD (GAL4 DNA binding domain). 

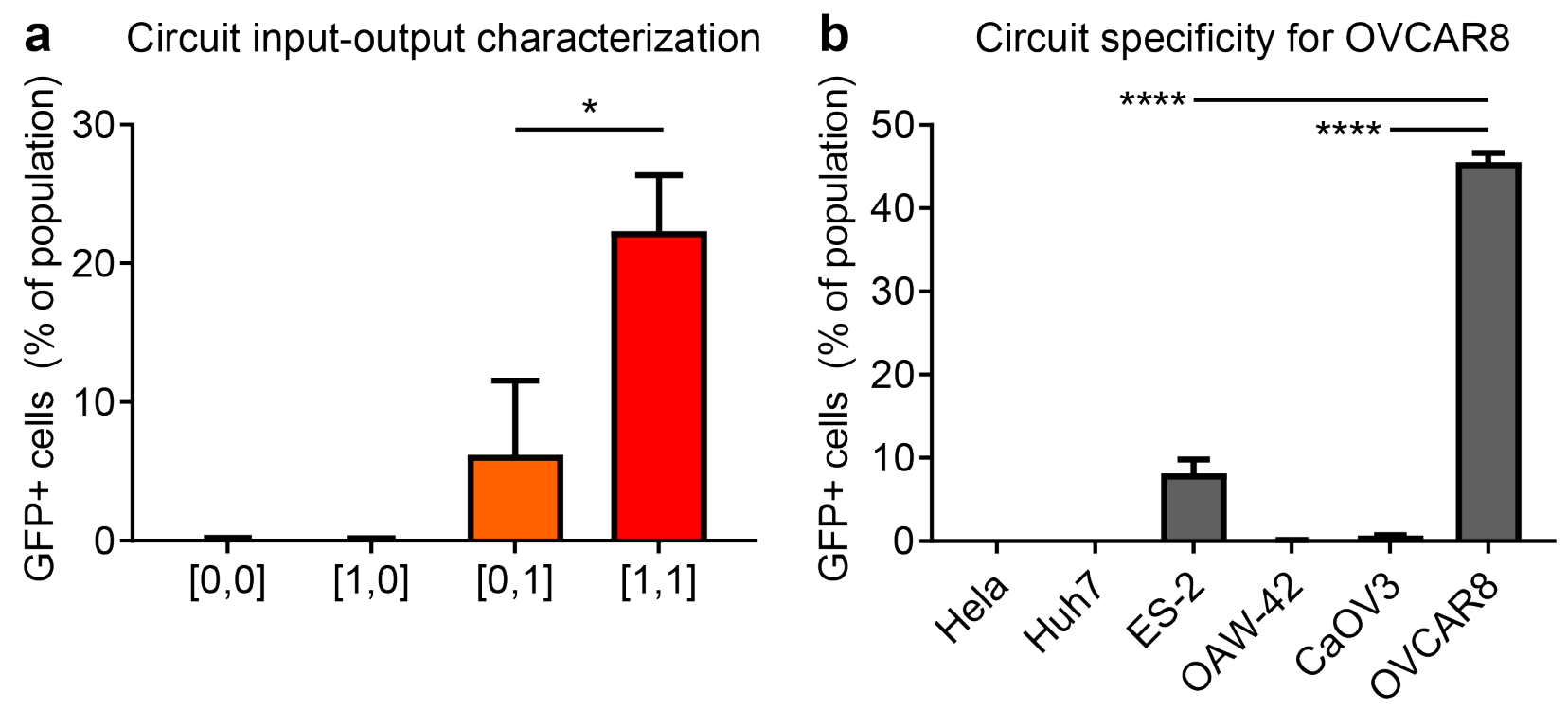

Figure S2. Output and specificity characterization of the synthetic circuit. A) OVCAR8 cells were transduced with the output virus carrying a membrane GFP expression cassette $([0,0])$, or the output virus with one of two input viruses $([1,0],[0,1])$, or both input viruses and the output virus $([1,1])$. After two days, cells were fixed for flow cytometry. Quantification of the percentage of the population positive for GFP for cells with different input and output configurations. Mean \pm s.d.; $\mathrm{N}=3$; unpaired two-tailed t-test, $* P=0.0140$. B) Human ovarian cancer cell lines (ES-2, OAW-42, CaOV-3, and OVCAR8), a hepatocarcinoma cell line (Huh7), and a cervical cancer cell line (Hela) with both input viruses and the output virus with GFP output $([1,1])$. After two days, cells were fixed for flow cytometry analysis. Quantification of the percentage of the population positive for GFP for different cell lines. Mean \pm s.d.; $\mathrm{N}=3$; unpaired two-tailed t-test, $* * * * P<0.0001$. 

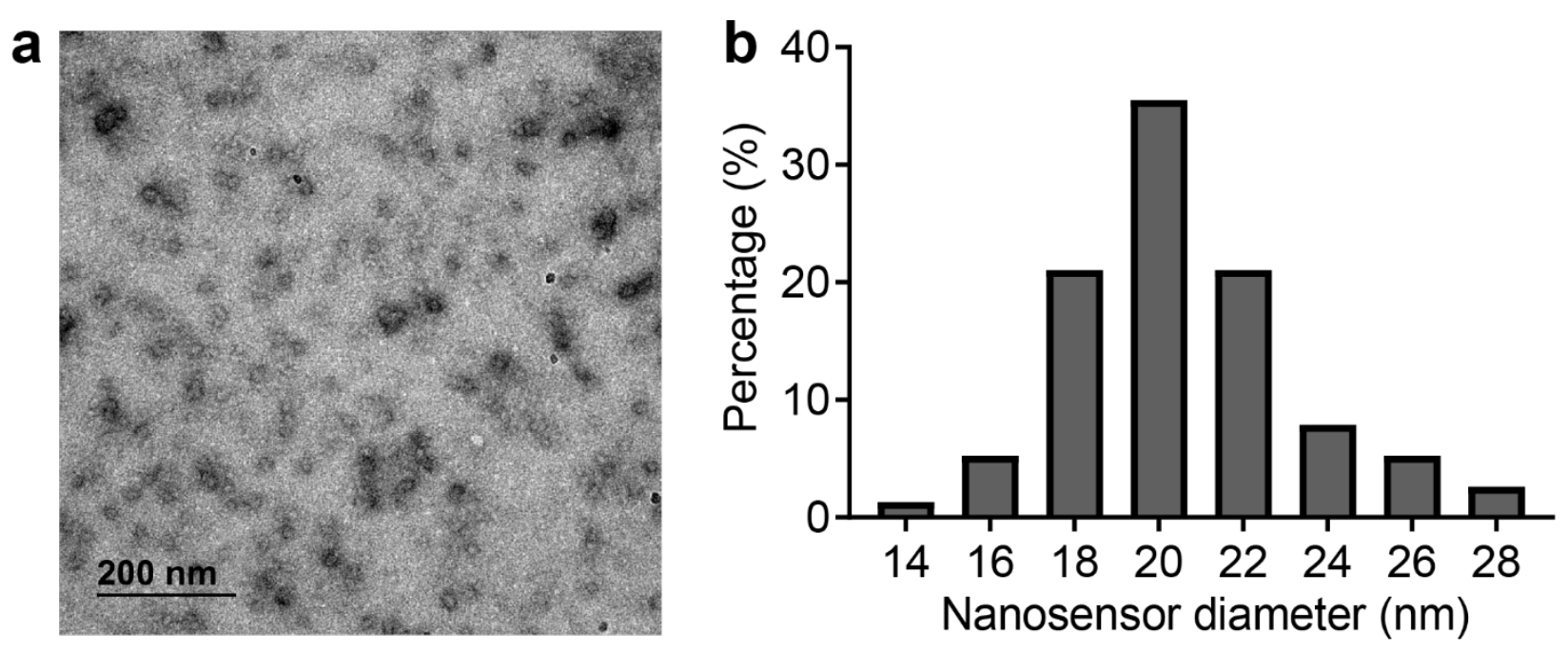

Figure S3. Characterization of the PEG-based nanosensor. A) TEM image of the TEV-sensitive nanosensor. Scale bar: $200 \mathrm{~nm}$. B) The histogram shows results of the size analysis from TEM images of the nanosensors (mean diameter, $20.6 \pm 2.8 \mathrm{~nm}$ (s.d.), $\mathrm{N}=76$ particles). 


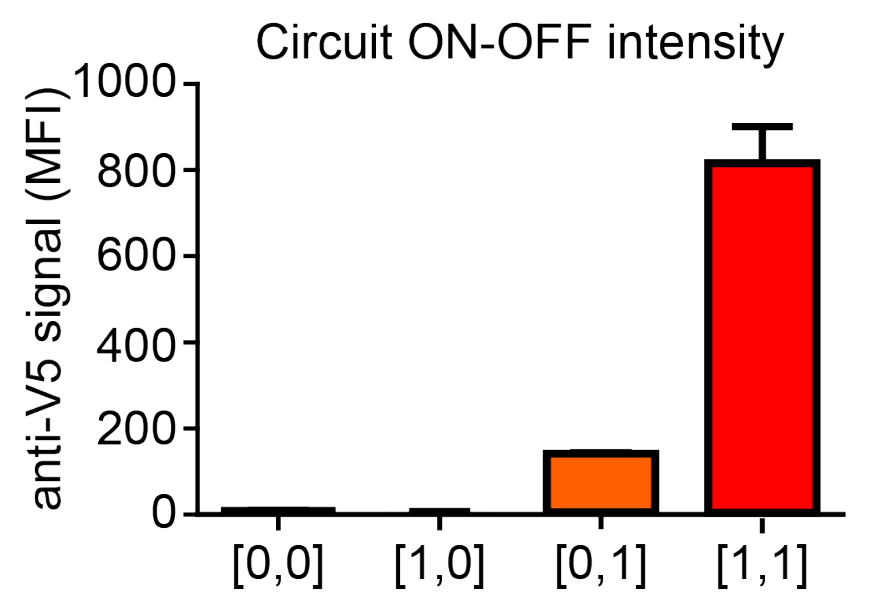

Figure S4. In vitro characterization of circuit ON-OFF ratio. OVCAR8 cells were transduced either with the output virus carrying the TEV protease expression cassette $([0,0])$, or the output virus with either of two input viruses $([1,0],[0,1])$, or the output virus and both of the input viruses $([1,1])$. After two days, cells were fixed to visualize the presence of the TEV protein output by flow cytometry analysis via detection of the V5 epitope tag (Fig. 4b). Quantification of flow cytometry analysis of mean GFP fluorescent intensity with different input and output configurations, as gated by $[0,0]$ state. Mean \pm s.d.; $\mathrm{N}=3$. 


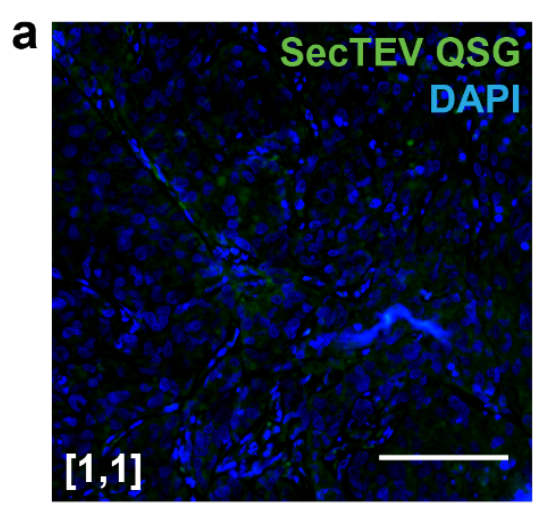

Liver

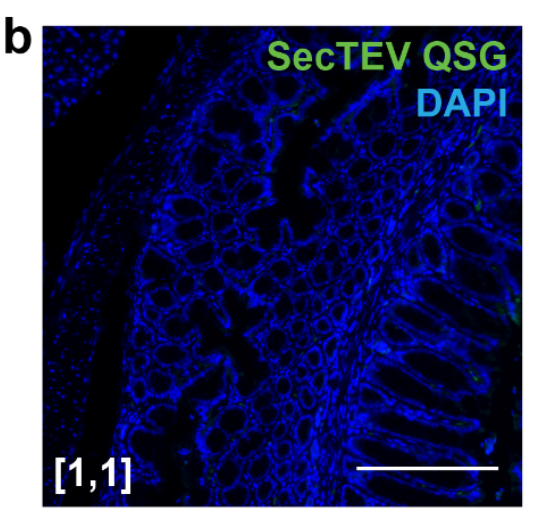

Intestine

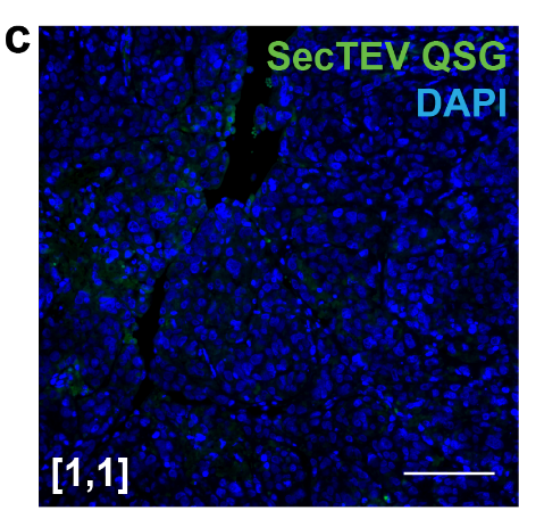

i.p. stroma

Figure S5. Circuit-produced TEV abundance in healthy tissues. 3-4 week old nude mice were i.p. injected with 5 million luciferase-expressing OVCAR8 cells. After 4 weeks, mice were i.p. injected with the complete synthetic circuit, corresponding to the [1,1] state. For mice with positive TEV staining in tumor nodules, TEV protease abundance in regions surrounding the tumor, including the liver (A), the intestine (B), and the surrounding intraperitoneal stroma (C), was assessed by staining with an anti-V5 antibody. Scale bar: $100 \mu \mathrm{m}$. 

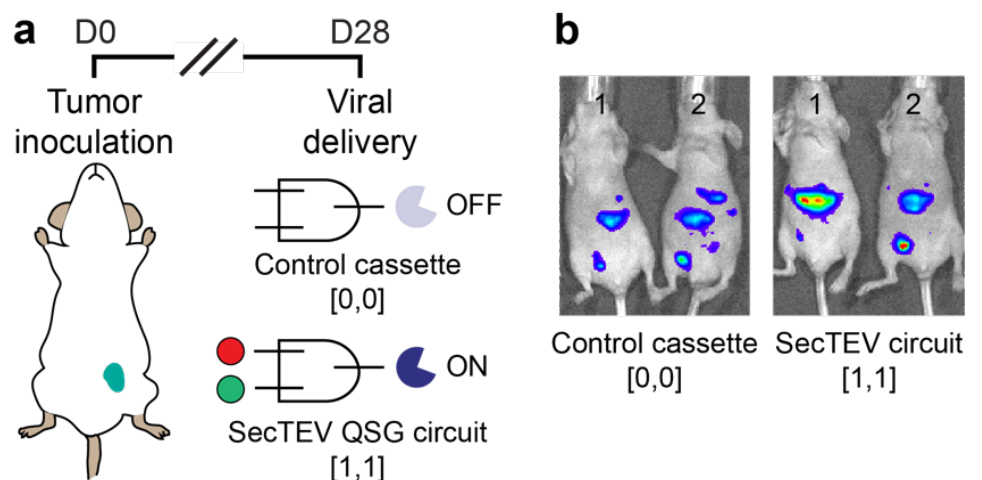

c

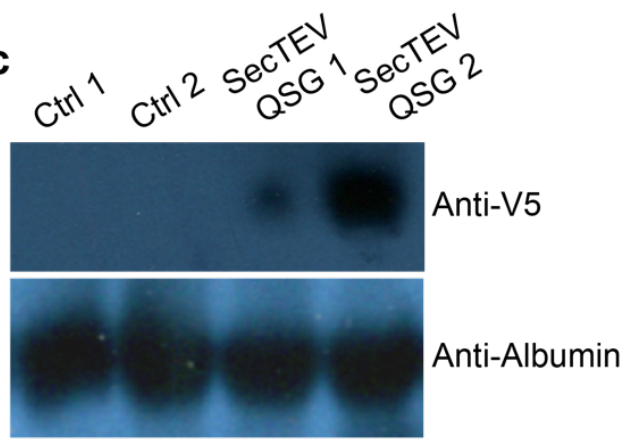

Figure S6. Detection of circuit-produced TEV protease in the blood following in vivo transduction. A) 3-4 week old nude mice were i.p. injected with 5 million luciferase-expressing OVCAR 8 cells. After 4 weeks, the mice were i.p. injected with the control cassette alone (Ctrl, $[0,0])$ or the complete SecTEV QSG synthetic circuit (SecTEV QSG, [1,1]). End stage blood collection was conducted through cardiac puncture two weeks post viral transduction. B) Representative IVIS images of OVCAR8-luciferase-inoculated mice transduced with either the control cassette or the complete SecTEV QSG synthetic circuit. C) Abundance of secreted TEV protease in the serum from viral-transduced OVCAR8 tumor-bearing mice, as measured via Western blotting against the V5 epitope tag present on circuit-produced SecTEV QSG. 


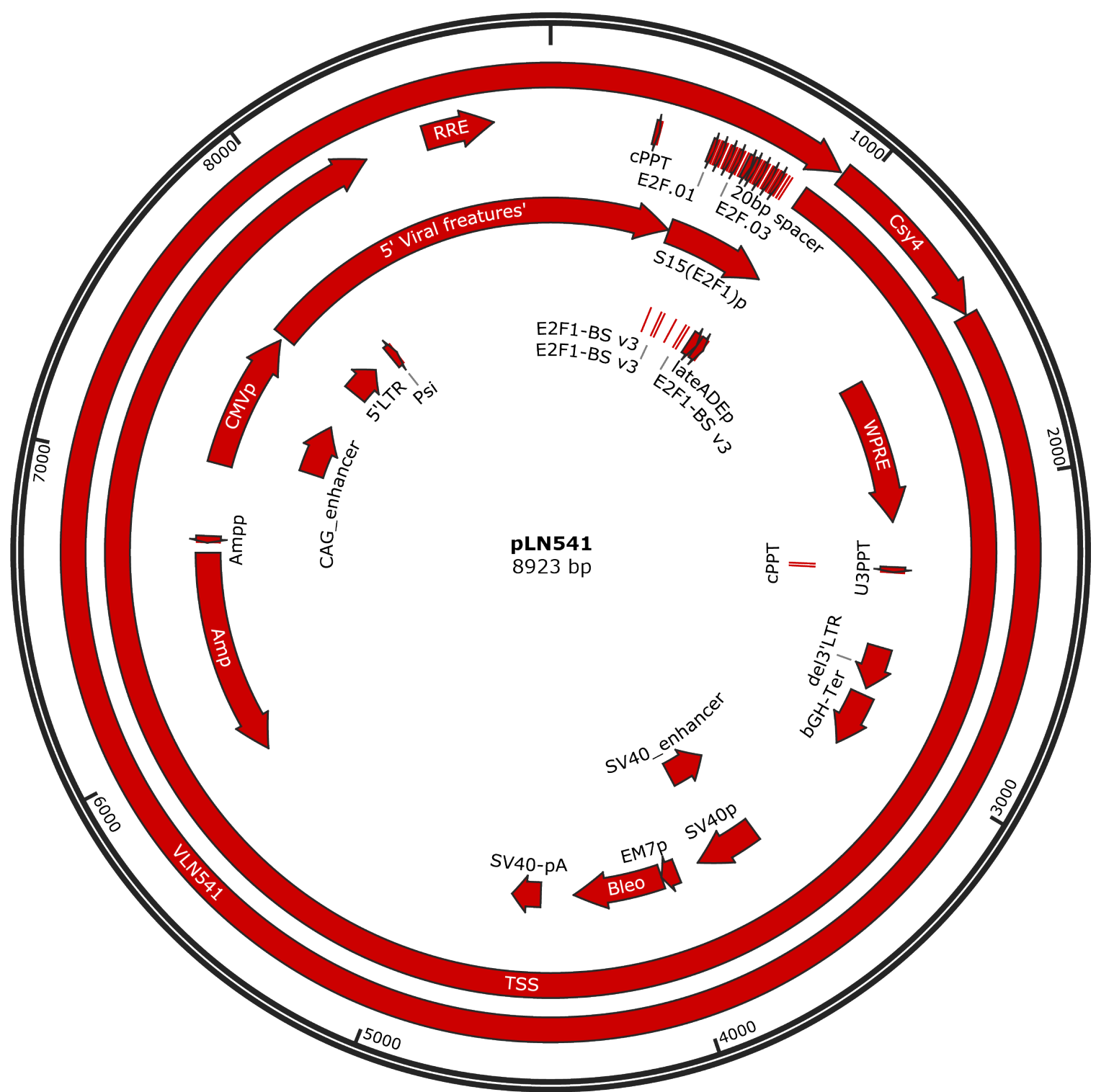

Figure S7. Plasmid map for Input 1 (Csy4) circuit component. 


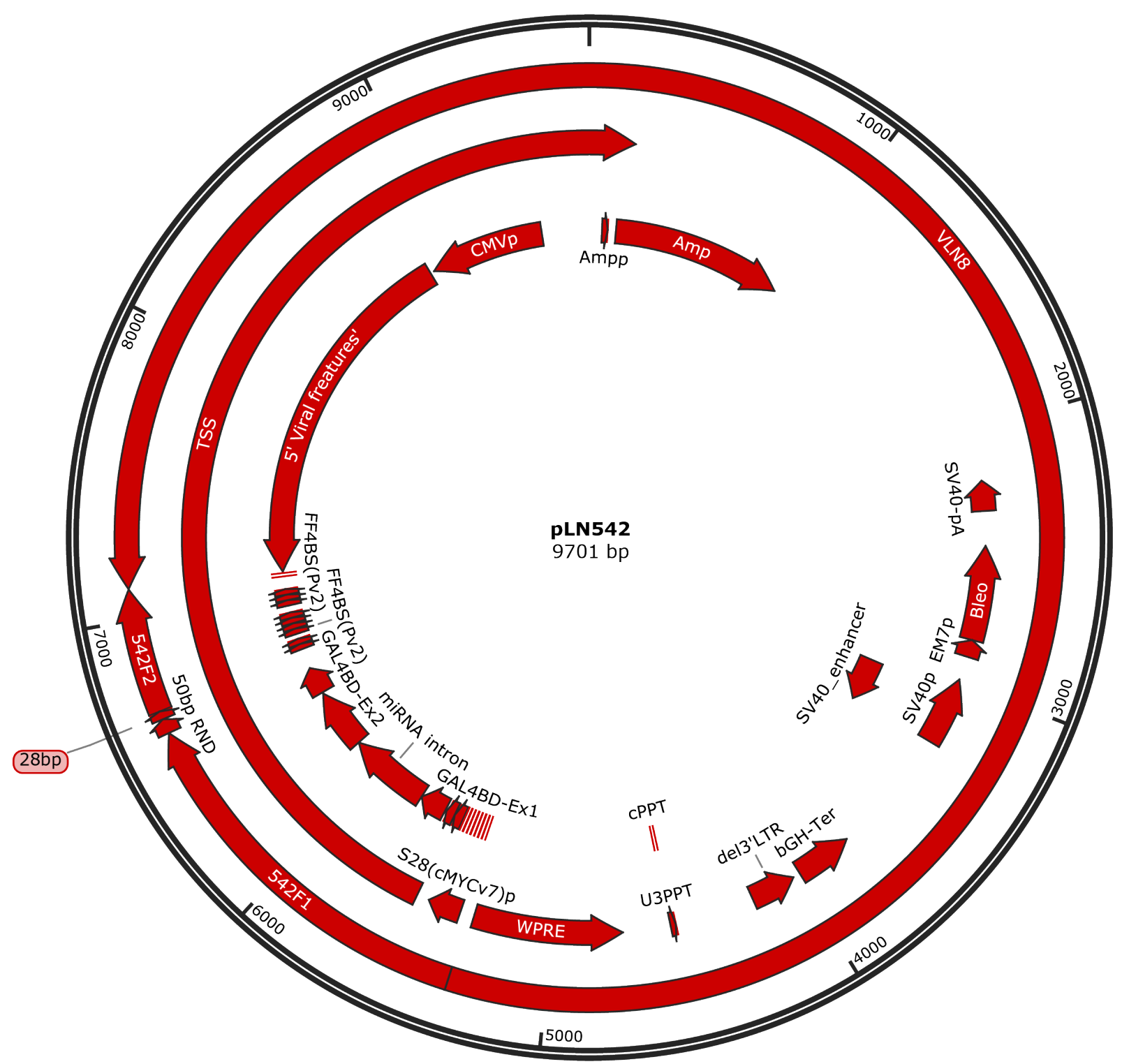

Figure S8. Plasmid map for Input 2 (GAD) circuit component. 


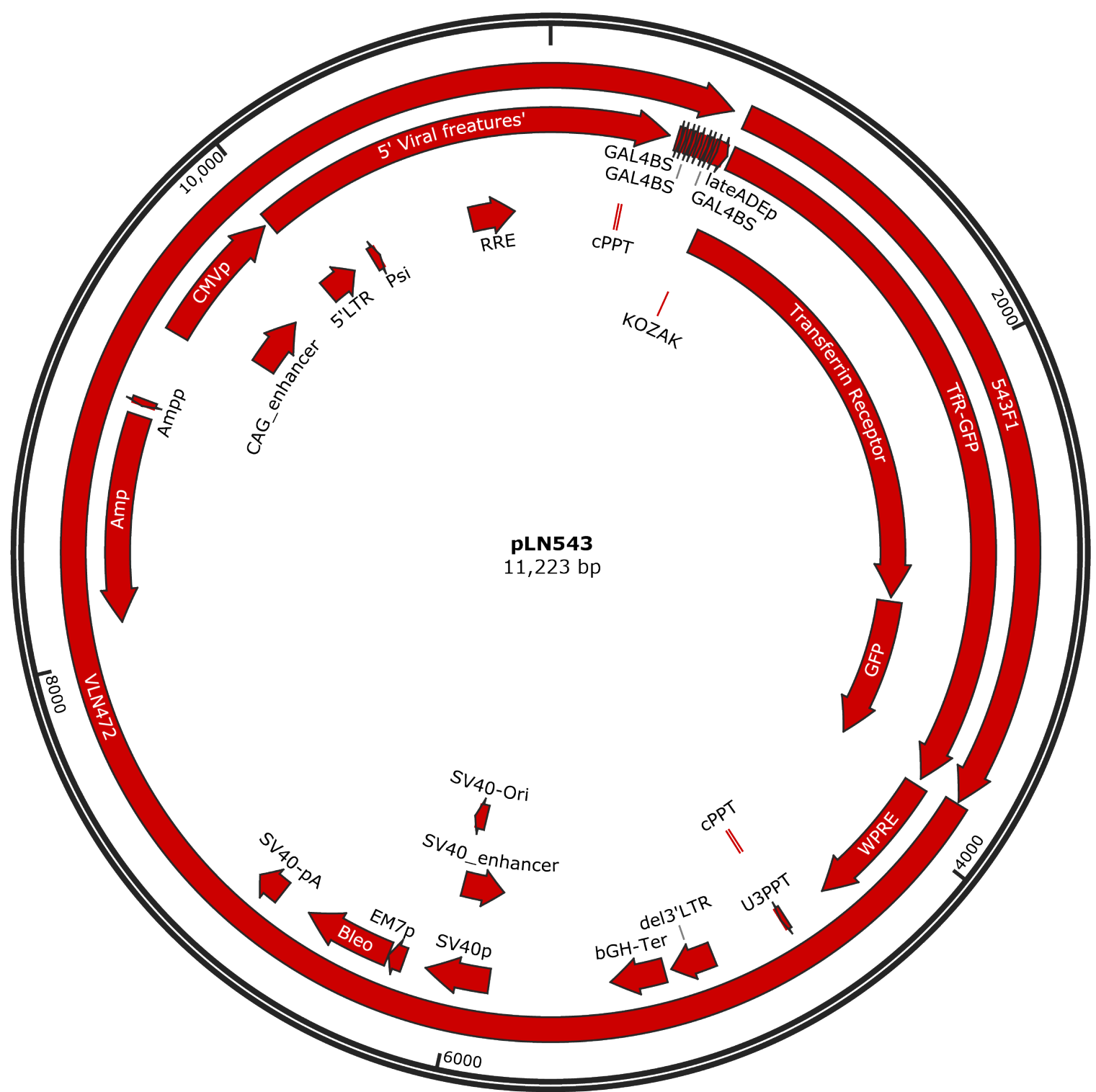

Figure S9. Plasmid map for output cassette with hTFR-GFP. 


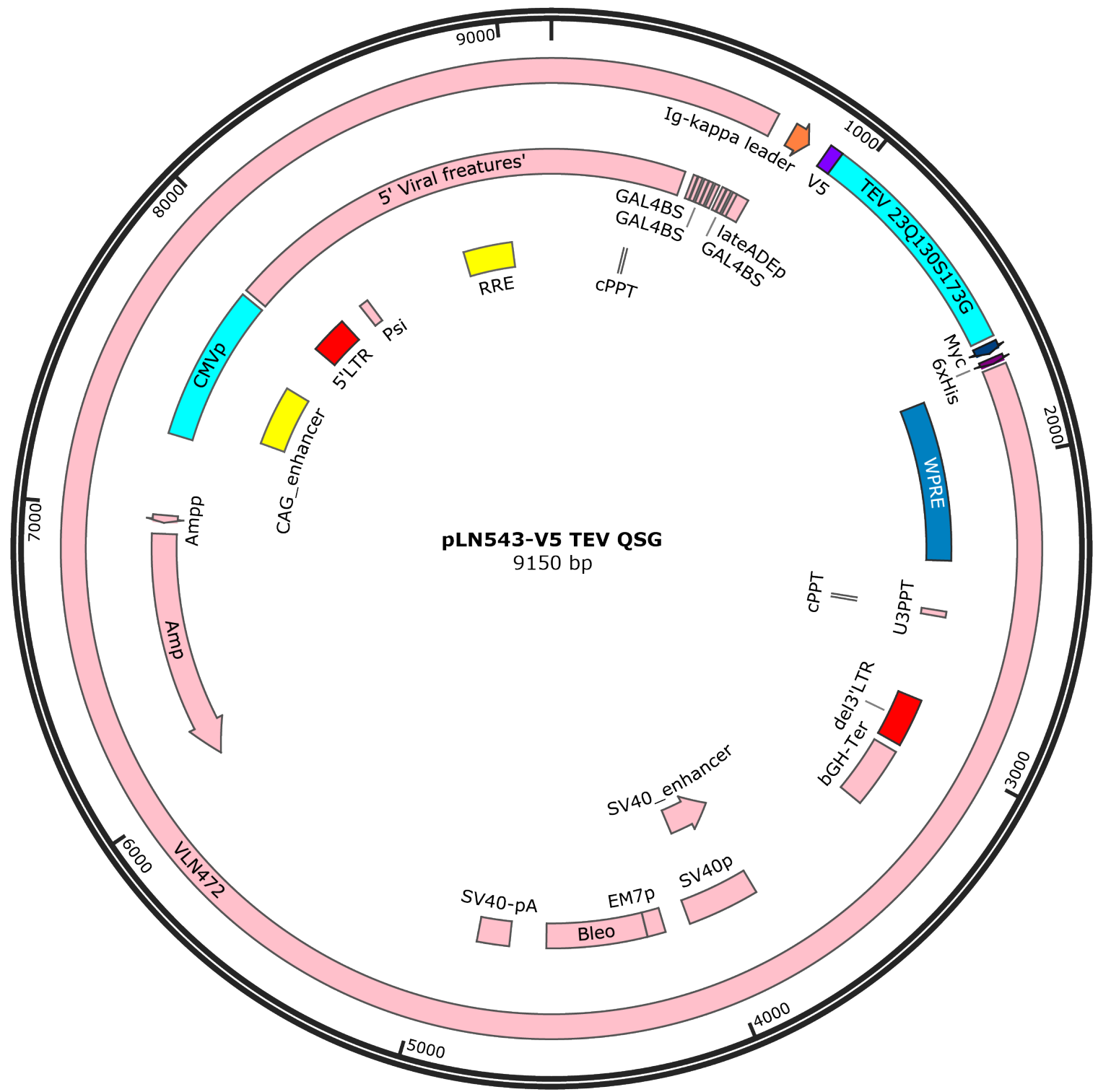

Figure S10. Plasmid map for output cassette with SecTEV-QSG. 
Table S1. Plasmid parts and their sequences.

\begin{tabular}{|c|c|}
\hline Name & Sequence \\
\hline$S(E 2 F 1) P$, promoter & $\begin{array}{l}\text { ATAAGCTTGATATCGCGGCCGCCCTGCACCTGCAGGTACGCGCGAAAAC } \\
\text { TGAGCGCGCCAAAATCGCTGAGCGCGAAACGGACGGGGCGGGAAGACT } \\
\text { TACGCGCGAAAACTGGTCGCGCCAAAGAGGCTGAGCGCGAAACGTCCG } \\
\text { GGGCGGGAAGCGAATTCTGCCCTACTGACACTGCCTGCCGTACGCGCGA } \\
\text { AAACTGAGCGCGCCAAAATCGCTGAGCGCGAAACGGACGGGGCGGGAA } \\
\text { GACTTACGCGCGAAAACTGGTCGCGCCAAAGAGGCTGAGCGCGAAACG } \\
\text { TCCGGGGCGGGAAGCGAATATCCGCGGCGCGCCAGACGCTAGCGGGGG } \\
\text { GCTATAAAAGGGGGTGGGGGCGTTCGTCCTCACTCTAGATCTGCGATCT } \\
\text { AAGTAAGCTTGGCATTCCGGTACTGTTGGTAAACCAGCT }\end{array}$ \\
\hline Csy4 & $\begin{array}{l}\text { GACCACTATCTGGACATCAGACTGAGGCCCGATCCTGAGTTCCCT } \\
\text { CCCGCCCAGCTGATGAGCGTGCTGTTTGGAAGCTGCATCAGGCT } \\
\text { CTGGTCGCCCAAGGCGGAGACAGAATCGGCGTGTCCTTCCCCGAC } \\
\text { CTGGACGAGTCCCGGAGTCGCCTGGGCGAGCGGCTGAGAATCCA } \\
\text { CGCCAGCGCAGACGATCTGCGCGCCCTGCTGGCCCGGCCTTGGCT } \\
\text { GGAGGGCCTGCGGGATCATCTGCAGTTTGGCGAGCCCGCCGTGGT } \\
\text { GCCACACCCAACACCCTACCGCCAGGTGAGCCGCGTGCAGGCCA } \\
\text { AGTCAAATCCCGAGAGACTGCGGCGGAGGCTGATGAGGCGACAT } \\
\text { GATCTGAGCGAGGAGGAGGCCAGAAAGAGAATCCCCGACACAGT } \\
\text { GGCCAGAGCCCTGGATCTGCCATTTGTGACCCTGCGGAGCCAGAG } \\
\text { CACTGGCCAGCATTTCAGACTGTTCATCAGACACGGGCCCCTGCA } \\
\text { GGTGACAGCCGAGGAGGGCGGATTTACATGCTATGGCCTGTCTAA } \\
\text { AGGCGGCTTCGTGCCCTGGTTC }\end{array}$ \\
\hline$S(c M y c) P$, promoter & $\begin{array}{l}\text { GTCCGCCACGTGCGCGACAGTCCGCCACGTGCGCGACTGGTCCGC } \\
\text { CACGTGCGCGACCGTCCGCCACGTGCGCGACCGGTCCGCCACGTG } \\
\text { CGCGACCGTCCGCCACGTGCGCGACGTGTCCGCCACGTGCGCG }\end{array}$ \\
\hline GAL4BD Ex1, of GAD & $\begin{array}{l}\text { ATGAAGCTACTGTCTTCTATCGAACAAGCATGCGATATTTGCCGA } \\
\text { CTTAAAAAGCTCAAGTGCTCCAAAGAAAAACCGAAGTGCGCCAA } \\
\text { GTGTCTGAAGAACAACTGGGAGTGTCGCTACTCTCCCAAAACCAA } \\
\text { AAG }\end{array}$ \\
\hline GAL4BD Ex2, of GAD & $\begin{array}{l}\text { GTCTCCGCTGACTAGGGCACATCTGACAGAAGTGGAATCAAGGCT } \\
\text { AGAAAGACTGGAACAGCTATTTCTACTGATTTTTCCTCGAGAAGA } \\
\text { CCTTGACATGATTTTGAAAATGGATTCTTTACAGGATATAAAAGC } \\
\text { ATTGTTAACAGGATTATTTGTACAAGATAATGTGAATAAAGATGC } \\
\text { CGTCACAGATAGATTGGCTTCAGTGGAGACTGATATGCCTCTAAC } \\
\text { ATTGAGACAGCATAGAATAAGTGCGACATCATCATCGGAAGAGA } \\
\text { GTAGTAACAAAGGTCAAAGACAGTTGACTGTATCG }\end{array}$ \\
\hline 28bp hairpin & GTTCACTGCCGTATAGGCAGCTAAGAAA \\
\hline miRNA intron & $\begin{array}{l}\text { GTAAGTGTGCTCGCTTCGGCAGCACATATACTATGTTGAATGAGG } \\
\text { CTTCAGTACTTTACAGAATCGTTGCCTGCACATCTTGGAAACACTT } \\
\text { GCTGGGATTACTTCTTCAGGGACTTCTTAACCCAACAGAAGGCTC } \\
\text { GAGAAGGTATATTGCTGTTGACAGTGAGCGCCGCTTGAAGTCTTT } \\
\text { AATTAAATAGTGAAGCCACAGATGTATTTAATTAAAGACTTCAAG } \\
\text { CGGTGCCTACTGCCTCGGACTTCAAGGGGCTAGAATTCAAGGGGC } \\
\text { TACTTTAGGAGCAATTATCTTGTTTACTAAAACTGAATACCTTGCT } \\
\text { ATCTCTTTGATACATTTTTACAAAGCTGAATTAAAATGGTATAAA } \\
\text { TTAAATCACTTTTTTCAATTGTACTAACTTCGAGTCTTCTTTTTTTT } \\
\text { TTTCACAG }\end{array}$ \\
\hline
\end{tabular}




\begin{tabular}{|c|c|}
\hline $\begin{array}{l}\text { VP16-AD, activation } \\
\text { domain of GAD }\end{array}$ & $\begin{array}{l}\text { TCGACGGCCCCCCCGACCGATGTCAGCCTGGGGGACGAGCTCCAC } \\
\text { TTAGACGGCGAGGACGTGGCGATGGCGCATGCCGACGCGCTAGA } \\
\text { CGATTTCGATCTGGACATGTTGGGGGACGGGGATTCCCCGGGTCC } \\
\text { GGGA }\end{array}$ \\
\hline $\begin{array}{l}\text { FF4BS(Pv2), 9x for } \\
\text { miRNABS }\end{array}$ & GCACCGCTTGAAGTCTTTAATTAAATAC \\
\hline GAL4BS & GGAGTACTGTCCTCCG \\
\hline $\begin{array}{l}\text { Transferrin receptor, } \\
\text { for hTFR-GFP }\end{array}$ & $\begin{array}{l}\text { ATGGATCAAGCTAGATCAGCATTCTCTAACTTGTTTGGTGGAGAA } \\
\text { CCATTGTCATATACCCGGTTCAGCCTGGCTCGGCAAGTAGATGGC } \\
\text { GATAACAGTCATGTGGAGATGAAACTTGCTGTAGATGAAAAGA } \\
\text { AAATGCTGACAATAACACAAAGGCCAATGTCACAAAACCAAAAA } \\
\text { GGTGTAGTGGAAGTATCTGCTATGGGACTATTGCTGTGATCGTCT } \\
\text { TTTTCTTGATTGGATTTATGATTGGCTACTTGGGCTATTGTAAAGG } \\
\text { GGTAGAACCAAAAACTGAGTGTGAGAGACTGGCAGGAACCGAGT } \\
\text { CTCCAGTGAGGGAGGAGCCAGGAGAGGACTTCCCTGCAGCACGT } \\
\text { CGCTTATATTGGGATGACCTGAAGAGAAAGTTGTCGAGAAACT } \\
\text { GGACAGCACAGACTTCACCAGCACCATCAAGCTGCTGAATGAAA } \\
\text { ATTCATATGTCCCTCGTGAGGCTGGATCTCAAAAAGATGAAAATC } \\
\text { TTGCGTTGTATGTTGAAAATCAATTTCGTGAATTAAACTCAGCA } \\
\text { AAGTCTGGCGTGATCAACATTTTGTTAAGATTCAGGTCAAAGACA } \\
\text { GCGCTCAAAACTCGGTGATCATAGTTGATAAGAACGGTAGACTTG } \\
\text { TTTACCTGGTGGAGAATCCTGGGGGTTATGTGGCGTATAGTAAGG } \\
\text { CTGCAACAGTTACTGGTAAACTGGTCCATGCTAATTTGGTACTA } \\
\text { AAAAAGATTTTGAGGATTTATACACTCCTGTGAATGGATCTATAG } \\
\text { TGATTGTCAGAGCAGGGAAAATCACCTTTGCAGAAAAGGTTCA } \\
\text { AATGCTGAAAGCTTAAATGCAATTGGTGTGTTGATATACATGGAC } \\
\text { CAGACTAAATTCCCATTGTTAACGCAGAACTTTCATTCTTTGGAC } \\
\text { ATGCTCATCTGGGGACAGGTGACCCTTACACACCTGGATTCCCTT } \\
\text { CCTTCAATCACACTCAGTTTCCACCATCTCGGTCATCAGGATTGCC } \\
\text { TAATATACCTGTCCAGACAATCTCCAGAGCTGCTGCAGAAAAGCT } \\
\text { GTTTGGGAATATGGAAGGAGACTGTCCCTCTGACTGGAAAACAG } \\
\text { ACTCTACATGTAGGATGGTAACCTCAGAAAGCAAGAATGTGAAG } \\
\text { CTCACTGTGAGCAATGTGCTGAAAGAGATAAAAATTCTTAACATC } \\
\text { TTTGGAGTTATTAAAGGCTTTGTAGAACCAGATCACTATGTTGTA } \\
\text { GTTGGGGCCCAGAGAGATGCATGGGGCCCTGGAGCTGCAAAATC } \\
\text { CGGTGTAGGCACAGCTCTCCTATTGAAACTTGCCCAGATGTCTC } \\
\text { AGATATGGTCTTAAAAGATGGGTTTCAGCCCAGCAGAAGCATTAT } \\
\text { CTTTGCCAGTTGGAGTGCTGGAGACTTTGGATCGGTTGGTGCCAC } \\
\text { TGAATGGCTAGAGGGATACCTTTCGTCCCTGCATTTAAAGGCTTT } \\
\text { CACTTATATTAATCTGGATAAAGCGGTTCTTGGTACCAGCAACTT } \\
\text { CAAGGTTTCTGCCAGCCCACTGTTGTATACGCTTATTGAGAAAAC } \\
\text { AATGCAAAATGTGAAGCATCCGGTTACTGGGCAATTTCTATATCA } \\
\text { GGACAGCAACTGGGCCAGCAAAGTTGAGAAACTCACTTTAGACA } \\
\text { ATGCTGCTTTCCCTTTCCTTGCATATTCTGGAATCCCAGCAGTTTC } \\
\text { TTTCTGTTTTTCGAGGACACAGATTATCCTTATTTGGGTACCACC } \\
\text { ATGGACACCTATAAGGAACTGATTGAGAGGATTCCTGAGTTGAAC } \\
\text { AAAGTGGCACGAGCAGCTGCAGAGGTCGCTGGTCAGTTCGTGATT } \\
\text { AAACTAACCCATGATGTTGAATTGAACCTGGACTATGAGAGGTAC } \\
\text { AACAGCCAACTGCTTTCATTTGTGAGGGATCTGAACCAATACAGA } \\
\text { GCAGACATAAAGGAAATGGGCCTGAGTTTACAGTGGCTGTATTCT } \\
\text { GCTCGTGGAGACTTCTTCCGTGCTACTTCCAGACTAACAACAGAT }\end{array}$ \\
\hline
\end{tabular}




\begin{tabular}{|c|c|}
\hline & $\begin{array}{l}\text { TTCGGGAATGCTGAGAAAACAGACAGATTTGTCATGAAGAAACT } \\
\text { CAATGATCGTGTCATGAGAGTGGAGTATCACTTCCTCTCTCCCTA } \\
\text { CGTATCTCCAAAAGAGTCTCCTTTCCGACATGTCTTCTGGGGCTCC } \\
\text { GGCTCTCACACGCTGCCAGCTTTACTGGAGAACTTGAAACTGCGT } \\
\text { AAACAAAATAACGGTGCTTTTAATGAAACGCTGTTCAGAAACCA } \\
\text { GTTGGCTCTAGCTACTTGGACTATTCAGGGAGCTGCAAATGCCCT } \\
\text { CTCTGGTGACGTTTGGGACATTGACAATGAGTTT }\end{array}$ \\
\hline GFP, for hTFR-GFP & $\begin{array}{l}\text { ATGGTGAGCAAGGGCGAGGAGCTGTTCACCGGGGTGGTGCCCAT } \\
\text { CCTGGTCGAGCTGGACGGCGACGTAAACGGCCACAAGTTCAGCG } \\
\text { TGTCCGGCGAGGGCGAGGGCGATGCCACCTACGGCAAGCTGACC } \\
\text { CTGAAGTTCATCTGCACCACCGGCAAGCTGCCCGTGCCCTGGCCC } \\
\text { ACCCTCGTGACCACCCTGACCTACGGCGTGCAGTGCTTCAGCCGC } \\
\text { TACCCCGACCACATGAAGCAGCACGACTTCTTCAAGTCCGCCATG } \\
\text { CCCGAAGGCTACGTCCAGGAGCGCACCATCTTCTTCAAGGACGAC } \\
\text { GGCAACTACAAGACCCGCGCCGAGGTGAAGTTCGAGGGCGACAC } \\
\text { CCTGGTGAACCGCATCGAGCTGAAGGGCATCGACTTCAAGGAGG } \\
\text { ACGGCAACATCCTGGGGCACAAGCTGGAGTACAACTACAACAGC } \\
\text { CACAACGTCTATATCATGGCCGACAAGCAGAAGAACGGCATCAA } \\
\text { GGTGAACTTCAAGATCCGCCACAACATCGAGGACGGCAGCGTGC } \\
\text { AGCTCGCCGACCACTACCAGCAGAACACCCCCATCGGCGACGGC } \\
\text { CCCGTGCTGCTGCCCGACAACCACTACCTGAGCACCCAGTCCGCC } \\
\text { CTGAGCAAAGACCCCAACGAGAAGCGCGATCACATGGTCCTGCT } \\
\text { GGAGTTCGTGACCGCCGCCGGGATCACTCTCGGCATGGACGAGCT } \\
\text { GTACAAGCGGACCGTC }\end{array}$ \\
\hline SecTEV QSG & $\begin{array}{l}\text { GGCGAGAGCCTCTTCAAGGGACCCAGGGACTACAACCCCATTAG } \\
\text { CAGCACCATCTGCCACCTCACCCAGGAGTCCGACGGCCATACCAC } \\
\text { CAGCCTGTATGGAATCGGCTTTGGACCTTCATCATCACCAACAA } \\
\text { GCATCTGTTTCGGCGGAACAACGGCACCCTGCTGGTGCAGAGCCT } \\
\text { GCATGGCGTGTTCAAGGTCAAGAACACAACTACCCTCCAGCAGC } \\
\text { ACCTGATCGATGGAAGAGACATGATCATCATCCGGATGCCAAAA } \\
\text { GACTTTCCACCTTTTCCCCAAAAACTCAAATTCCGGGAGCCTCAG } \\
\text { AGAGAAGAGCGGATCTGTCTGGTGACCACAAATTTCAGACAAA } \\
\text { AAGCATGAGCAGCATGGTGTCCGATACAAGCAGCACATTCCCATC } \\
\text { CAGCGATGGCATCTTCTGGAAACATTGGATTCAGACAAAAGATG } \\
\text { GCCAGTGCGGAAGCCCACTCGTCTCCACTAGAGACGGGTTCATCG } \\
\text { TGGGGATTCACAGCGCTAGCAATTTTGGAAATACCAATAATTACT } \\
\text { TTACATCCGTCCCAAAGAACTTCATGGAGCTGCTGACAAACCAGG } \\
\text { AAGCACAGCAGTGGGTGAGCGGCTGGAGGCTGAACGCCGATTCC } \\
\text { GTCCTGTGGGGCGGCCACAAGGTCTTCATGGTGAAGCCCGAAGA } \\
\text { GCCTTTCCAGCCAGTGAAGGAGGCCACTCAACTGATGAAC }\end{array}$ \\
\hline
\end{tabular}

